\title{
Glassy Behavior of Electrons as a Precursor to the Localization Transition
}

\author{
V. Dobrosavljević ${ }^{a}$ A. A. Pastor ${ }^{a}$ \\ ${ }^{a}$ Department of Physics and National High Magnetic Field Laboratory \\ Florida State University, 1800E Paul Dirac Dr., Tallahassee, FL 32310, USA
}

\begin{abstract}
A theoretical model is presented, describing the glassy freezing of electrons in the vicinity of disorder driven metal-insulator transitions. Our results indicate that the onset of glassy dynamics should emerge before the localization transition is reached, thus predicting the existence of an intermediate metallic glass phase between the normal metal and the insulator.
\end{abstract}

Key words: Metal-insulator transition, glassy behavior

PACS: 73.50.Td, 64.70.Pf, 71.23.-k

\section{Introduction}

Disorder-driven metal-insulator transitions (MITs) in strongly correlated electronic systems continue to demonstrate fascinating behaviors that remain to be elucidated. The competition of electron-electron interactions and disorder is most pronounced in the insulator, where one generally expects the existence of a large number of low energy configurations of the electronic density, leading to glassy behavior, metastability, and slow relaxation. Far from the transition, the kinetic energy of the electrons is negligible and one can utilize a classical picture, as pioneered in early work by Efros and Shklovskii [2]. The precise role of glassy dynamics is more difficult to assess closer to the MIT, where quantum fluctuations due to electronic mobility become important. In recent work [3], we have developed a formalism capable of describing such glassy behavior of electrons both in the classical limit and in presence of quantum fluctuations.

\footnotetext{
* Corresponding author: V. Dobrosavljević, fax. 850-644-5393

Email address: vlad@magnet.fsu.edu (V. Dobrosavljević).
} 
Here we extend this approach to incorporate Anderson localization effects [4], which prove to have a dramatic effect on the stability of the glass phase.

The following physical picture emerges from our study: Anderson localization leads to the formation of bound electronic states, thus suppressing quantum fluctuations introduced by mobile electrons. However, we find that glassy behavior is not eliminated as soon as the electrons become delocalized. Very close to the MIT the electronic mobility remains very small, thus limiting the size of quantum fluctuations needed to overcome barriers between metastable states. An intermediate metallic glass phase is predicted, separating the insulator and the normal metal.

\section{Model}

We consider the simplest microscopic model capable of describing the interplay of glassy behavior and Anderson localization effects. It is given by a one-band tight-binding model of spinless electrons at half filling, with nearest-neighbor hopping $t_{i j}$, interacting by infinite range inter-site interactions $V_{i j}$, in presence of random site energies $\varepsilon_{i}$. For technical simplicity, these interactions are chosen also to be random [5], with zero mean and variance $<V_{i j}^{2}>=V^{2} / N$, where $N$ is the number of sites in the systems. In the following, we briefly outline the formalism that we use; the details will be presented elsewhere [6]. We first formally average over random interactions using the replica method [3], which generates a four-density interaction term. This term is then decoupled by introducing Hubbard-Stratonovich fields, and the resulting action takes the form $S_{\text {eff }}=S_{\text {el }}+S_{\text {int }}$, where

$$
\begin{aligned}
S_{e l}=\int_{o}^{\beta} d \tau \sum_{a} \sum_{<i j>} c_{i}^{\dagger a}(\tau)\left[\left(\partial_{\tau}+\varepsilon_{i}\right) \delta_{i j}-t_{i j}\right] c_{i}^{a}(\tau), \\
S_{\text {int }}=V \sum_{i} \int_{o}^{\beta} d \tau \int_{o}^{\beta} d \tau^{\prime}\left[\sum_{a} \tilde{Q}_{i}^{a a}\left(\tau, \tau^{\prime}\right) \delta n_{i}^{a}(\tau) \delta n_{i}^{a}\left(\tau^{\prime}\right)\right. \\
\left.+\sum_{a<b} Q_{i}^{a b}\left(\tau, \tau^{\prime}\right) \delta n_{i}^{a}(\tau) \delta n_{i}^{b}\left(\tau^{\prime}\right)\right] .
\end{aligned}
$$

We have used standard functional integration over replicated Grassmann fields, where $a=1, \ldots, n(n \rightarrow 0)$ are the replica indices [3]. Here, the operators $\delta n_{i}^{a}(\tau)=\left(c_{i}^{\dagger a}(\tau) c_{i}^{a}(\tau)-1 / 2\right)$ represent the density fluctuations from half filling.

In the classical limit $\left(t_{i j}=0\right)$, these equations can be solved exactly due to the infinite range of the interactions $V_{i j}$, but once finite-range hopping 
is introduced, the resulting electronic degrees of freedom generate additional effective nonlocal interactions between density modes, and we have to resort to approximations. A mean-field treatment of the interactions is then provided by evaluating the $Q$-fields in the saddle-point approximation, which also makes it possible to identify the replica symmetry breaking instability used to establish the onset of glassy ordering [3].

The resulting instability criterion takes the form

$$
1=V^{2}<\frac{1}{N} \sum_{i j}\left(\chi_{i j}\right)^{2}>_{d i s}
$$

Here, the non-local compressibilities $\chi_{i j}$ are defined by

$$
\chi_{i j}=\frac{1}{\beta} \int_{o}^{\beta} d \tau \int_{o}^{\beta} d \tau^{\prime}\left[<\delta n_{i}^{a}(\tau) \delta n_{j}^{a}\left(\tau^{\prime}\right)>_{o}-<\delta n_{i}^{a}(\tau)>_{o}<\delta n_{j}^{a}\left(\tau^{\prime}\right)>_{o}\right],
$$

where the quantum averages $\langle\cdots\rangle_{o}$ are taken with respect to the full replicasymmetric action $S_{\text {eff }}$ evaluated at the saddle-point, and $\langle\cdots\rangle_{\text {dis }}$ indicates the average with respect to disorder.

Further simplification is obtained [3] by concentrating on the limit of large disorder, where to leading order one can evaluate the compressibilities by setting $V=0$ in $S_{\text {eff }}$. The calculation then reduces to calculating the compressibilities with respect to a system of noninteracting electrons in presence of disorder. Physically, this calculation amounts to focusing on electrons in the vicinity of the Anderson transition, and examining the leading perturbation introduced by turning on inter-site interactions $V_{i j}$. The crucial insight is obtained by observing that in contrast to the average compressibilities $<\chi_{i j}>_{d i s}$, the quantities $\left\langle\left(\chi_{i j}\right)^{2}>_{\text {dis }}\right.$ actually diverge at $T=0$ at the Anderson transition. We immediately conclude that Anderson localization introduces a singular perturbation to the stability of the glass phase, as anticipated in Ref. [3]. In addition, we note that the sum in Eq. (3) runs over positive definite terms, so that a lower bound to the size of the glass phase is obtained by retaining only the local $(i=j)$ term. We have examined this quantity using several different approaches to Anderson localization, but the simplest closed form expression can be obtained by using the recently developed typical medium theory [7].

We have used a simple semi-circular model density of states [3] in presence of a uniform distribution of random site energies of width $W$, and have done calculations as a function of the disorder strength $W$ and temperature $T$. We find that $\left\langle\left(\chi_{i i}\right)^{2}>_{d i s} \sim\left(W_{c}-W\right)^{-1}\right.$ indeed blows up at $T=0$ precisely at the Anderson transition, which for this model occurs at $W_{c} \approx 1.36 B$, where $B$ is the electronic bandwidth. As a result, the instability criterion for the 
emergence of the glass phase is satisfied before the Anderson transition is reached, for any finite value of the interaction $V$. To illustrate our findings, we present the resulting phase diagram for $W / V=2$, as a function of $W$ and $T$ in Fig. 1. Note that the "kink" in the glass transition boundary at $W=W_{c}$ (full line) reflects the crudeness of our treatment of localization, which ignores the fact that inelastic scattering effects will tend to delocalize the electrons at any finite temperature. These inelastic effects can be accounted for by keeping the interaction terms in $S_{\text {eff }}$, but these corrections will be investigated in more detail elsewhere. To illustrate the qualitative effects of such inelastic scattering, we phenomenologically introduce a temperature-dependent scattering rate of the form $\eta=A T$. The resulting modification of the phase boundary for the choice $A=0.1$ is presented by a dotted line, leading to the rounding of the kink, as expected.

\section{Discussion}

Our results strongly suggest that the onset of glassy dynamics should be considered as a precursor to disorder-driven metal-insulator transitions. Physically, as the system approaches localization the electrons are barely mobile, allowing for even moderate interactions to induce meta-stability and glassy freezing. We do not expect this glassy freezing to have a direct effect on the average conductivity, but it should be more readily identified by examining the slowing down of the electron dynamics. In particular, as the glassy phase is entered, we expect large enhancements for the low frequency components of the conductivity noise spectra, reflecting slow transitions between emerging metastable states. An interesting question relates to the the size of the proposed metallic glass phase as a function of disorder. This intermediate phase is expected to be extremely small for very strong disorder, since in this limit the effect of the interactions leading to glassy behavior should be very modest. But what should be expected for very weak disorder but strong interactions? In the limit where $r_{s}=V / E_{F}>>1$, insulating behavior can emerge due to Wigner crystallization, even in absence of disorder. As the Wigner crystal is approached, we expect the local compressibility to be strongly reduced, reflecting strong short range order induced by electronic correlations. As a result, the possibility for many electronic configurations is again impaired, suppressing glassy ordering. We thus expect that the best chance to observe the metallic glass phase is for moderately disordered systems with $W / V \sim 1$. Remarkably, very recent experiment on a two-dimensional electron gas in silicon seems to support this picture. In this material, clear evidence for an intermediate metallic glass phase has been discovered for low mobility samples [8], but not in high-mobility devices. In latter systems, the emergence of glassy dynamics seems to coincide with the MIT [9], as one would expect for systems closer to 
a (disordered) Wigner crystal.

This work was supported by the NSF grant DMR-9974311 and the National High Magnetic Field Laboratory. We thank S. Bogdanovich, J. Jaroszyński, and D. Popović for useful and stimulating discussions.

\section{References}

[1] N. F. Mott, 1990 Metal-Insulator Transitions (London: Taylor and Francis, 1990).

[2] A. L. Efros and B. I. Shklovskii, J. Phys. C8 L49(1975).

[3] A. A. Pastor and V. Dobrosavljević, Phys. Rev. Lett. 83, 4642 (1999).

[4] P. W. Anderson, Phys. Rev. 109, 1498 (1958)

[5] In recent work [3], we have shown that in presence of random site energies, such random interactions are generated by renormalization even if one starts with purely repulsive interactions in the bare Hamiltonian. Similar conclusions have been obtained numerically in: E. R. Grannan and C. C. Yu, Phys. Rev. Lett. 71, 3335 (1993).

[6] V. Dobrosavljević and A. A. Pastor, in preparation.

[7] V. Dobrosavljević and A. A. Pastor, preprint, cond-mat/0106282.

[8] S. Bogdanovich and D. Popović, proceedings of EP2DS14, Physica E (2002).

[9] J. Jaroszyński, D. Popović, and T. M. Klapwijk, proceedings of EP2DS14, Physica E (2002). 


\section{Figure captions}

Figure 1

Phase diagram for spinless interacting electrons in presence of disorder, as a function of temperature $T$ and the Fermi energy $E_{F}$, expressed in units of the disorder strength $W$. Results are presented for moderate interaction strength $W / V=2$. The glass transition boundary is shown, in absence of inelastic scattering (full line) and in presence of an inelastic scattering rate $\eta=0.1 T$ (dotted line). Note that the glassy phase emerges before the metal-insulator transition (MIT) is reached (heavy dot). The (glassy) Anderson insulator is shown with a heavy full line (at $T=0)$. 


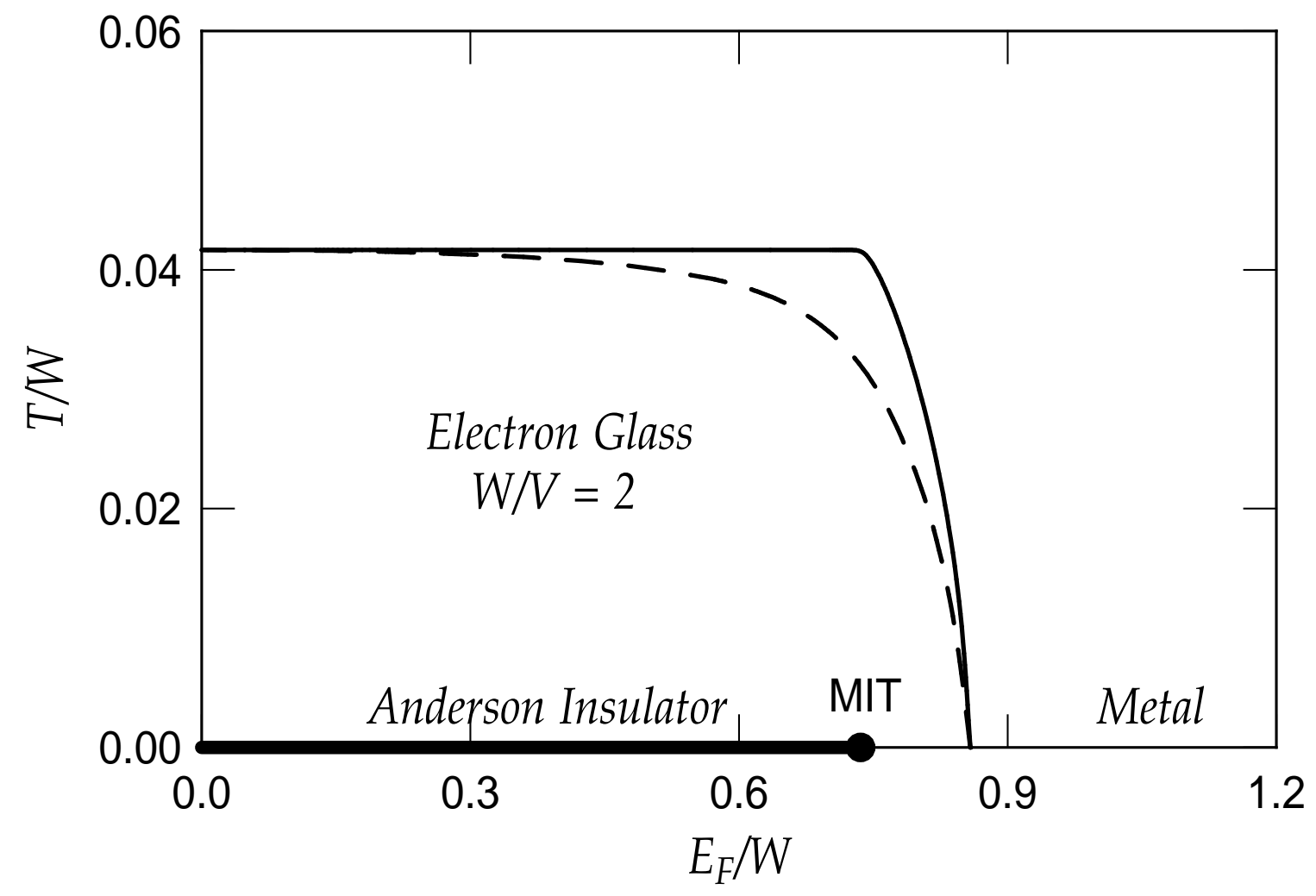

